\title{
Purest Ever Example-Based Machine Translation: detailed presentation and assessment
}

\author{
Y. Lepage (yves.lepage@atr.jp) \\ ATR, Keihanna, 619-0288 Kyōto, Japan \\ E. Denoual (etienne.denoual@atr.jp)* \\ ATR, Keihanna, 619-0288 Kyōto, Japan \& \\ GETA-CLIPS-IMAG, université Joseph Fourier, 38041 Grenoble Cedex 9, France
}

\begin{abstract}
We have designed, implemented and assessed an EBMT system that can be dubbed the 'purest ever built': it strictly does not make any use of variables, templates or patterns, does not have any explicit transfer component, and does not require any preprocessing or training of the aligned examples. It only uses a specific operation, proportional analogy, that implicitly neutralises divergences between languages and captures lexical and syntactical variations along the paradigmatic and syntagmatic axes without explicitly decomposing sentences into fragments. Exactly the same genuine implementation of such a core engine was evaluated on different tasks and language pairs. To begin with, we compared our system on two tasks of a previous MT evaluation campaign to rank it among other current state-of-the-art systems. Then, we illustrated the 'universality' of our system by participating in a recent MT evaluation campaign, with exactly the same core engine, for a wide variety of language pairs. Finally, we studied the influence of extra data like dictionaries and paraphrases on the system performance.
\end{abstract}

Keywords: Example-based machine translation, proportional analogies, divergences across languages.

Abbreviations: MT - machine translation; EBMT - example-based machine translation; mWER - multiple word error rate

${ }^{*}$ The research reported here was supported in part by a contract with the Japanese National Institute of Information and Communications Technology entitled "A study of speech dialogue translation technology based on a large corpus". We are particularly indebted to Prof. C. Boitet for his many comments on an earlier version of the draft that considerably helped to improve clarity. Thanks also to the reviewers who pointed out some errors in the draft. Both authors are currently with the Japanese National Institute of Information and Communications Technology $(\mathrm{NiCT})$.

(C) 2006 Kluwer Academic Publishers. Printed in the Netherlands. 


\section{Introduction}

In contrast to some other approaches to machine translation, namely statistical machine translation, which do not view linguistic data as specific data, we believe that natural language tasks are specific because their data are specific. The object of this paper is to show that the use of a specific operation, namely proportional analogy in our present proposal, is profitable in terms of trading off the preprocessing time of the data and the quality of the results.

We present a novel example-based machine translation system, which relies entirely on proportional analogy. An appealing feature of our system is that it requires no training whatsoever: the data are simply loaded into memory at start-up and they are not preprocessed in any way. This is a definite advantage over techniques that require intensive preprocessing. Another consequence is that it can be applied directly to any language pair for which there is sufficient available data.

We evaluated our system on the tasks of the IWSLT 2004 evaluation campaign (AKIBA et al., 2004) in the Japanese-English and ChineseEnglish Unrestricted Data tracks. This evaluation showed that, at that time, our system would have positioned itself among the top systems for these tracks. We also demonstrated the ubiquity of the system by having exactly the same translation engine participating in all possible language pairs of the IWSLT 2005 evaluation campaign. As the data loaded in memory at start-up constitute the only translation knowledge of our system, we also inspected the influence of these data on translation results.

After this introduction, we shall detail in section 2 the claims on which our proposal relies. In section 3 , we shall sketch the core process of the translation engine, make some remarks on its features and illustrate it with scholar and real examples. Section 4 shall give the theoretical foundations of the proposal. Section 5 shall detail a number of experiments done, their results and some comparison with other stateof-the-art systems. The remaining section shall discuss and provide a conclusion over the features of the system and the obtained results.

\section{The claims}

\subsection{Dealing With the Specificity of Linguistic Data}

Trivially, any linguistic datum belongs to one specific natural language that constitutes a 'system' in the Saussurian sense of the term. A consistent consequence is to process linguistic data using operations 
that specifically capture this systematicity. This systematicity appears at best in commutations exhibited in proportional analogies like those of the following examples:

\begin{tabular}{|c|c|c|c|}
\hline $\begin{array}{l}\text { It walks } \\
\text { across the } \\
\text { street. }\end{array}$ & $\begin{array}{l}\text { It walked } \\
\text { across the } \\
\text { street. }\end{array}$ & $\begin{array}{l}\text { It floats } \\
\text { across the } \\
\text { river. }\end{array}$ & $\begin{array}{l}\text { It floated } \\
\text { across the } \\
\text { river. }\end{array}$ \\
\hline $\begin{array}{l}\text { I'd like to } \\
\text { open these } \\
\text { windows. }\end{array}$ & $\begin{array}{c}\text { Could you } \\
\text { open a } \\
\text { window? }\end{array}$ & $\begin{array}{l}\text { I'd like to } \\
\text { cash these } \\
\text { traveler's } \\
\text { checks. }\end{array}$ & $\begin{array}{c}\text { Could you } \\
\text { cash a } \\
\text { traveler's } \\
\text { check? }\end{array}$ \\
\hline
\end{tabular}

A proportional analogy is noted $A: B:: C: D$ in its general form and reads ' $A$ is to $B$ as $C$ is to $D$.' It is a logical predicate that necessarily takes four arguments, not less. We stress that four objects are required, because analogy is, alas, too often confused with mere similarity between two objects ${ }^{1}$. Because it is a logical predicate, when we take four sentences in a language to form a proportional analogy, the result is either true or false. For instance, the following proportional analogy is true,

$$
\begin{array}{cccc}
\text { It walks } & \text { It walked } & \text { It floats } & \text { It floated } \\
\text { across the : } & \text { across the :: } & \text { across the : } & \text { across the } \\
\text { street. } & \text { street. } & \text { river. } & \text { river. }
\end{array}
$$

while the following one is not:

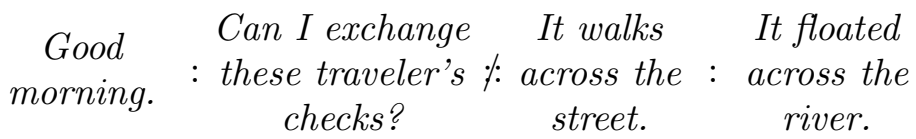

This is exactly the same thing as with proportions on numbers, the difference being that here we use sentences instead of numbers. Hence, for instance,

$$
\frac{5}{15}=\frac{10}{30} \quad \text { also written as } \quad 5: 15=10: 30
$$

is a valid proportion, whilst

$$
\frac{5}{15} \neq \frac{4}{8}
$$

is not.

The human interpretation of proportional analogies between sentences is that some pieces of the sentences commute with other pieces, 
so that human beings perceive it as a kind of parallel replacement. In the following example, we put such pieces into boxes. It is clear that such pieces are not necessarily words in general. In that example, although an English speaker would perceive an exchange of the word street with river, a shorter explanation in terms of total length of substrings exchanged is possible: str commutes with riv while et commutes with $r$. This may hurt the linguistic feeling, but the same speaker would easily admit that the $s$ in walks in the first sentence is exchanged with $e d \mathrm{n}$ the second sentence because $s$ and $e d$ are flexional morphemes affixed to verbal roots like walk.

$$
\begin{aligned}
& \text { It walks It walked It floats } \quad \text { It floated } \\
& \text { across the : across the :: across the : across the } \\
& \text { stret. } \quad \text { str et et. } \quad r i v e r . \quad r i v e r .
\end{aligned}
$$

From a global point of view, such commutations make paradigmatic and syntagmatic variations explicit and allow for lexical and syntactical variations, that ought to be exploited in a machine translation system to express different meanings. Indeed, any sentence in any language may be cast into a wide number of such proportional analogies that form a kind of meshwork around it. For instance, the sentence

\begin{tabular}{|c|c|c|c|c|c|c|}
\hline $\begin{array}{l}\text { It walks } \\
\text { across the } \\
\text { street. }\end{array}$ & : & $\begin{array}{l}\text { It walked } \\
\text { across the } \\
\text { street. }\end{array}$ & $::$ & $\begin{array}{l}\text { It floats } \\
\text { across the } \\
\text { river. }\end{array}$ & & $\begin{array}{l}\text { It floated } \\
\text { across the } \\
\text { river. }\end{array}$ \\
\hline It swam. & & $\begin{array}{l}\text { It swam } \\
\text { across the } \\
\text { river. }\end{array}$ & $::$ & It floated. & : & $\begin{array}{l}\text { It floated } \\
\text { across the } \\
\text { river. }\end{array}$ \\
\hline $\begin{array}{l}\text { They sw } \\
\text { in the se }\end{array}$ & & $\begin{array}{l}\text { It swam } \\
\text { across the } \\
\text { river. }\end{array}$ & $::$ & $\begin{array}{c}\text { They } \\
\text { floated in } \\
\text { the sea. }\end{array}$ & & $\begin{array}{l}\text { It } f \\
\text { acr } \\
r\end{array}$ \\
\hline
\end{tabular}

\section{It floated across the river.}

can be seen in the following analogies:

The first analogy can be interpreted as showing an opposition between the present and the past tenses in two different sentences. The second analogy can be interpreted as showing that a prepositional phrase may expand some verbs. Consequently, these two analogies can easily be labeled (present/past or w/o PP) and thus located according to some linguistically accepted categorisation. But this is not the case 


\begin{tabular}{|l|l|l|l|}
\hline $\begin{array}{l}\text { I like Japanese } \\
\text { food. }\end{array}$ & $\begin{array}{l}\text { I prefer Japan- } \\
\text { ese food. }\end{array}$ & $\begin{array}{l}\text { I'd prefer Jap- } \\
\text { anese food. }\end{array}$ & $\begin{array}{l}\text { I feel like Jap- } \\
\text { anese food. }\end{array}$ \\
\hline $\begin{array}{l}\text { Do you like Ital- } \\
\text { an food? }\end{array}$ & $\begin{array}{l}\text { Do you feel like } \\
\text { Italian food? }\end{array}$ \\
\hline $\begin{array}{l}\text { I'd like Western } \\
\text { food. }\end{array}$ & $\begin{array}{l}\text { I'd prefer West- } \\
\text { ern food. }\end{array}$ & & \\
\hline $\begin{array}{l}\text { I like Chinese } \\
\text { food. }\end{array}$ & $\begin{array}{l}\text { I prefer Chinese } \\
\text { food. }\end{array}$ & (x) & \\
\hline $\begin{array}{l}\text { I like Italian } \\
\text { food. }\end{array}$ & $\begin{array}{l}\text { I prefer Italian } \\
\text { food. }\end{array}$ & $\begin{array}{l}\text { I feel like Mexi- } \\
\text { can food. }\end{array}$ \\
\hline $\begin{array}{l}\text { I like Mexican } \\
\text { food. }\end{array}$ & $\begin{array}{l}\text { feel like } \\
\text { seafood. }\end{array}$ \\
\hline $\begin{array}{l}\text { I like seafood. } \\
\text { I prefer seafood. }\end{array}$ & $\begin{array}{l}\text { I'd prefer West- } \\
\text { ern food. }\end{array}$ & \\
\hline $\begin{array}{l}\text { I like Western } \\
\text { food. }\end{array}$ &
\end{tabular}

Figure 1. An extract of a table that visualises several analogical relations between (simple) sentences extracted from our corpus.

in the third analogy, where it is difficult to find a label that would adequately characterise all oppositions involved (change in pronouns, singular / plural, different verbs, different circumstantial complements). The purpose of giving such examples of analogies is to show that, if it is usually understood that analogies exemplify well documented and described linguistic phenomena, actual occurrences in corpora are not always ideal examples of definite and well classified phenomena. In the discussion (see Section 7.5), we shall go back to the fact that what our method would lack to be more efficient is precisely consistent examples for well described phenomena, like: simple tense oppositions, singular / plural, affirmative / negative / interrogative sentences, etc., because they don't appear consistently in actual corpora.

In (Lepage and Peralta, 2004) we have shown how to automatically extract tables (or matrices) from a linguistic resource so as to visualise these meshworks: each cell in a table contains a sentence, and rectangles formed with four cells in the tables are proportional analogies. An example of such a table is given in Figure 1. It was obtained starting with the sentence I like Japanese food. The line with seafood and the other lines with Chinese, Italian, ... food show that word boundaries do not count as a specific place for commutations. From the table, it is also clear that new sentences may be added into the table into some of the cells that were left blank. For instance, the 
cell marked $(x)$ can be filled with the sentence I'd prefer Italian food. Such a sentence is obtained by solving an analogical equation in the same way as equations in proportions are solved. I'd prefer Italian food. can fill the cell marked $(x)$ because:

$$
\begin{array}{ccccc}
\text { I like } & \text { I'd prefer } & \text { I like } & & \text { I'd prefer } \\
\text { Japanese } & : & \text { Japanese } \\
\text { food. } & \text { food. } & \text { Italian } & \text { food. } &
\end{array} \quad \Rightarrow x=\begin{gathered}
\text { Italian } \\
\text { food. }
\end{gathered}
$$

in the same way as one writes:

$$
5: 15=10: x \Rightarrow x=30
$$

\subsection{Dealing With Divergences across languages}

Machine translation has specific problems to address: one of them, at the core of translation, is to tackle divergences across languages. Back in the early times of machine translation, the problem was pointed out by Vauquois and exemplified with the exchange of predicate arguments between French and English in the following famous example:

$$
\text { Elle }_{1} \text { lui }_{2} \text { plaît. } \quad \leftrightarrow \quad \mathrm{He}_{2} \text { likes her }{ }_{1} .
$$

Recent studies (DorR et al., 2002) confirm the importance of the phenomenon: on a sample of 19,000 sentences between English and Spanish it was estimated that one sentence in three presents divergences. A classification into five different types was proposed in (HABASH, 2002):

1. categorial divergences:

2. conflation:

3. structural divergence:

4. head swapping:

5. thematic divergence:

$$
\begin{array}{r}
\text { tener celos }(N) \leftrightarrow \text { to be jealous }(A) \\
\text { ir flotando } \leftrightarrow \text { to float } \\
\text { entrar en } N \leftrightarrow \text { to enter } N \\
\text { entrar corriendo } \leftrightarrow \text { to run in } \\
\text { me gustan uvas } \leftrightarrow \text { I like grapes }
\end{array}
$$

Let us examine an example of type 4 in further detail, i.e., the classical translation of a Spanish verb into an English preposition (AMORES and MorA, 1998) ${ }^{2}$. We can express the word-to-word correspondence by indexing words. The same index shows words in translation correspondence. The correspondence of atravesó with across through index 1 and that of flotando and floated through index 3 exemplify a translation divergence of type 4 , i.e., head swapping (see next page). 

1: Atravesó $_{\mathrm{V}}$
0: It
2: el río $\mathrm{N}$
$\leftrightarrow \quad \begin{aligned} & \text { 3: } \text { floated }_{\mathrm{V}} \\ & \text { 1: } \text { across }_{\text {prep. }} \\ & \text { 2: the river } \mathrm{N}\end{aligned}$
3: flotando particip.

To show that the complexity of divergences is often underestimated, let us stick with the above interpretation that Sp. atravesó would correspond to En. across. This kind of divergence easily gives rise to a configuration which is excluded by construction from Inversion Transduction Grammars. It is the 14th configuration in (WU, 1997, p. 386), that is called "inside-out matchings" by the author, who further claims that he has "been unable to find real examples in [his] data of constituent arguments undergoing "inside-out" transposition' (Chinese-English). However, the introduction of an adverb, which appears after the finite verb in Spanish, and before in English, leads to this very configuration:

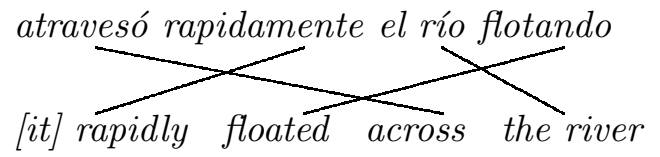

Let us now show that the view of word-to-word correspondence is all but partial and incomplete. Approaches that adopt the word as the unit of processing neglect the fact that corresponding pieces of information in different languages are indeed distributed over the entire strings and do not necessarily correspond to complete words. For this reason, the correspondence between words given in the example above is in fact not sufficiently detailed. Actually, the ending -ó of the first Spanish word accounts for the 3rd person singular past tense. So, not only does atravesó correspond to the English preposition across for its meaning, but, in addition, it also corresponds to another complete word in English (the pronoun $i t$ ), plus a portion of yet a third English word (the final ending -ed of floated). Consequently the following representation, where boxes show the correspondence, is more correct.

Atravesó el río flotando. $\leftrightarrow$ It floated across the river.

Unfortunately, again, this representation is partial, as it should be repeated for any word in the source language, or any word in the target language, or, even, put to the extreme, any sequence of characters in both the source and the target language.

If we wanted to drop the view where words correspond to words, we would logically have to deal with a finer grain than the word unit, 
and go to the level of characters. This would mean that, in order to express correspondences, we should compute the correspondences between characters or character strings. This approach is obviously risky as it would imply a combinatorial explosion in the number of correspondences to explore.

The following section will show that indeed, making such correspondences explicit can be avoided. The solution is achieved by stating the only necessary correspondence, the one that exists between two entire sentences in two different languages, as in:

$$
\text { Atravesó el río flotando. } \leftrightarrow \text { It floated across the river. }
$$

and relying on the structure of the languages to perform monolingual commutations instead of computing finer bilingual correspondences.

\subsection{Dealing With structures (meshworks of Proportional ANALOGIES)}

Following the previous idea that a sentence belongs to a meshwork of proportional analogies, any particular translation correspondence between two sentences belonging to two different languages should be viewed as a part of the global correspondence between the two languages at hand. The technique that we thus propose for automatic translation exploits the translation links that incidentally exist between sentences as part of the meshwork of proportional analogies found around them.

Figure 2 gives the example of the two following sentences taken as part of particular proportional analogies that correspond.

$$
\begin{aligned}
& \text { Could you cash a traveler's } \leftrightarrow \text { Vous pouvez m'échanger un } \\
& \text { check? } \leftrightarrow \text { chèque de voyage? }
\end{aligned}
$$

Here, we have chosen an example which is much more complex (at least in the French part) than the previous one with cross the river, so as to convince the reader of the difficulty of the puzzles that can be solved by our approach. The correspondence can only be established because each sentence in the lower part of the figure is a possible translation of the sentence above it in the upper part of the figure (indicated by a vertical arrow).

Another view of the scene is given by the parallelopiped of Figure 3. Each of the vertical planes of the parallelopiped resides in one and only one language. The one on the left is the English part, and the one on the right is the French part.

A consequence of this view is that the difficulty which is usually faced in translating between some particular languages partly vanishes 


\begin{tabular}{|c|c|c|c|}
\hline $\begin{array}{l}\text { I'd like to } \\
\text { open these } \\
\text { windows. }\end{array}$ & $\begin{array}{l}\text { Could you } \\
\text { open a } \\
\text { window? }\end{array}$ & $\begin{array}{l}\text { I'd like to } \\
\text { cash these } \\
\text { traveler's } \\
\text { checks. }\end{array}$ & $\begin{array}{c}\text { Could you } \\
\text { cash a } \\
\text { traveler's } \\
\text { check? }\end{array}$ \\
\hline$\uparrow$ & $\uparrow$ & $\uparrow$ & $\uparrow$ \\
\hline $\begin{array}{l}\text { Est-ce que } \\
\text { ces } \\
\text { fenêtres, là, : } \\
\text { je peux les } \\
\text { ouvrir? }\end{array}$ & $\begin{array}{c}\text { Est-ce que } \\
\text { vous pouvez } \\
\text { m'ouvrir } \\
\text { une } \\
\text { fenêtre? }\end{array}$ & $\begin{array}{c}\text { Ces } \\
\text { chèques de } \\
\text { voyage, là, } \\
\text { je peux les } \\
\text { échanger? }\end{array}$ & $\begin{array}{c}\text { Vous } \\
\text { pouvez } \\
\text { m'échanger } \\
\text { un chèque } \\
\text { de voyage? }\end{array}$ \\
\hline
\end{tabular}

Figure 2. Two proportional analogies in two different languages that correspond.

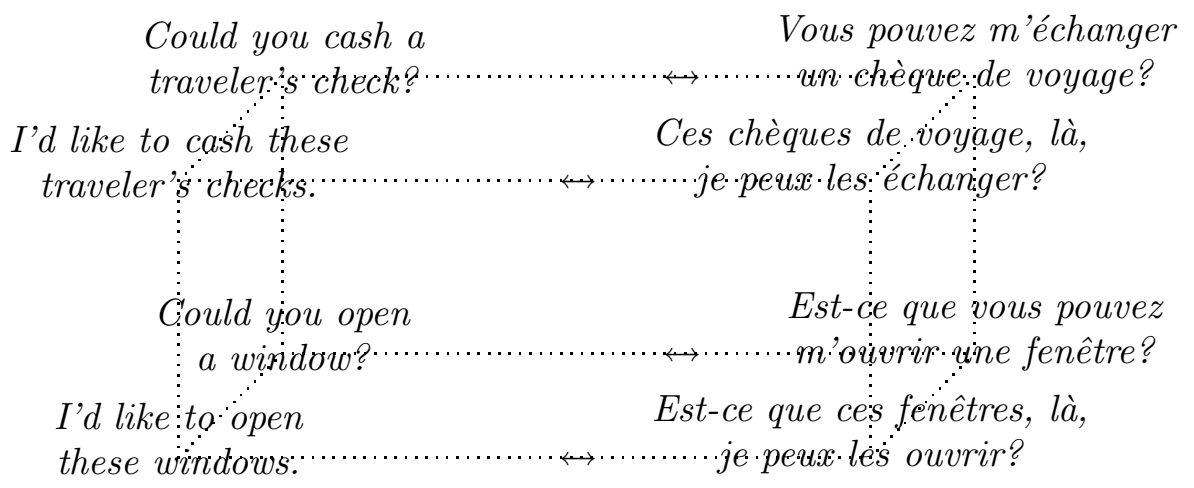

Figure 3. The parallelopiped: in each language, four sentences form a proportional analogy. There exist four translation relations between the sentences. This is just a geometrical representation of Figure 2.

(at least in theory!). The claim that it is costly to translate between some specific languages like, e.g., Japanese and English, relies indeed on the idea that translating would basically consist of rearranging, transforming, or decoding. For instance, (SumitA, 2003, p. 205) explicitely says: 'Because we have succeeded in J-to-E, one of the most difficult translation pairs, we have little concern about other pairs.'

However, to make a comparison with clothes, to localise what corresponds to the left shoulder of a shirt on, say, a jacket, one does not rearrange or transform the material of the shirt, i.e., one does not take material from the left shoulder of the shirt, unweave it, weave it back again in a different way, and then patch it somewhere on the jacket. Although this may sound strange, this is precisely what second generation MT systems actually do when they use lexical and structural 
transfer rules; and SMT systems (BRown et al., 1993) when they use lexicon models with distortion models (IBM models 4 and 5).

Rather, it is reasonable to point at the left shoulder of the jacket by looking at the general constitution of the jacket, and by following the different weaves and threads on the jacket to localise some point more precisely if needed, as the jacket is made of a different material from the shirt. Transposing to machine translation, the translation of a source sentence should be sought by relying on the paradigmatic and syntagmatic meshworks, i.e., by using the proportional analogies in the target language which correspond to the proportional analogies of the source language that involve the source sentence, until a corresponding sentence is obtained.

Basically, the method that we propose for translation is reminiscent of distributionalism (HARRIS, 1954): a sentence can be generated in the target language as long as there is a place for it in the meshwork of the target language. And a sentence of the source language can be translated only to the extent that it occupies some place in the meshwork of the source language. Consequently, expressions that are proper to a particular language, an example of which is the famous English idiom to kick the bucket, shall be translated in our proposed framework with no added difficulty than for any other usual sentence.
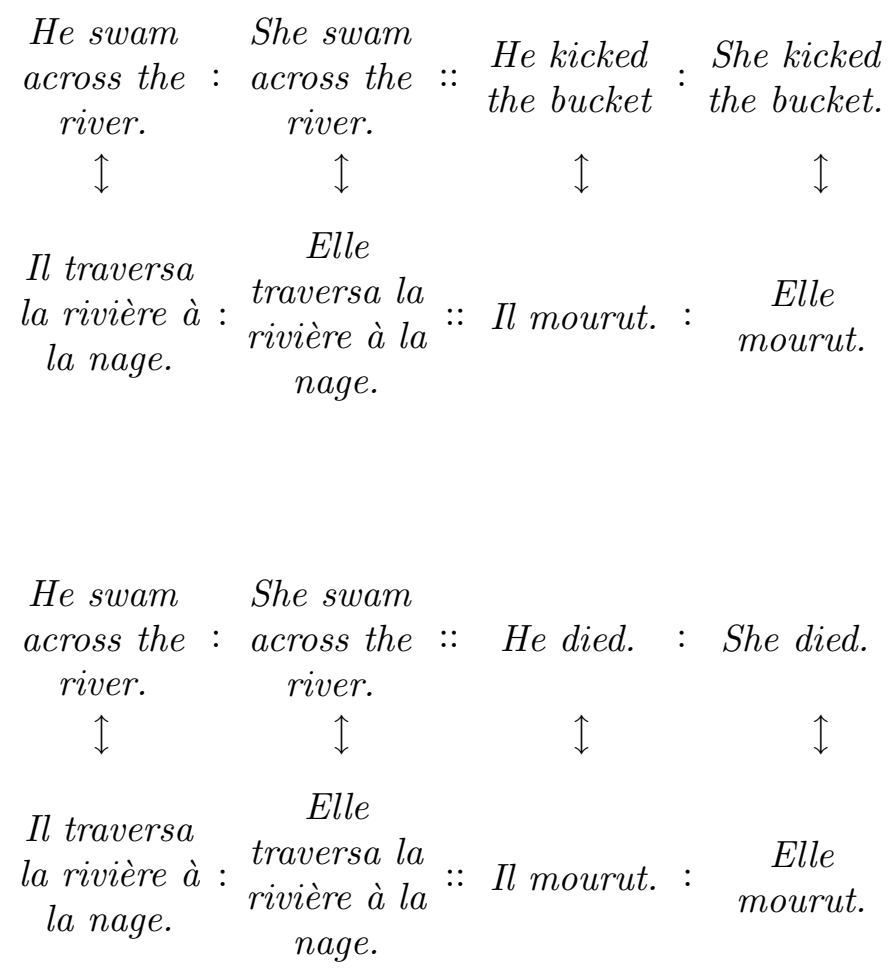


\section{Example-based machine translation (EBMT) by proportional analogy}

\subsection{The Algorithm}

The following gives the basic outline of the method we propose to perform the translation of an input sentence. Let us suppose that we have a corpus of aligned sentences in two languages (a bicorpus) at our disposal. Let $D$ be an input sentence to be translated into one or more target sentences $\widehat{D}$.

- Form all analogical equations with the input sentence $D$ and with all pairs of sentences $\left(A_{i}, B_{i}\right)$ from the source part of the bicorpus;

$$
A_{i}: B_{i}:: x: D
$$

- For those sentences that are solutions of the previous analogical equations, but that do not belong to the bicorpus, translate them using the present method recursively. Add them with their newly generated translations to the bicorpus;

- For those sentences $x=C_{i, j}$ that are solutions of the previous analogical equations (one analogical equation may yield several solutions) and which do belong to the bicorpus, do the following;

- Form all analogical equations with all possible target language sentences corresponding to the source language sentences (several target sentences may correspond to the same source sentence);

$$
{\widehat{A_{i}}}^{k}: \widehat{B}_{i}^{k}::{\widehat{C_{i, j}}}^{k}: y
$$

- Output the solutions $y={\widehat{D_{i, j}}}^{k, l}$ of the analogical equations as a translation of $D$, sorted by frequencies (different analogical equations may yield identical solutions).

\subsection{SOME REMARKS}

As the above algorithm may be misunderstood in various ways, it is necessary for us to make some remarks and clarify some points. This section serves this purpose.

Firstly, in order to avoid any misinterpretation where the method would be considered a method by decomposition where some breaking operation is involved, let us stress that $A_{i}, B_{i}$ and $D$ are sentences; 
they are not fragments of sentences. Sentences are not cut into pieces by the proposed method.

Secondly, pairs of sentences are retrieved to form an analogical equation with $D$. Consequently, speaking about analogous examples, does not make any sense in this framework. Again proportional analogy should not be mixed with mere similarity, and it should be stressed that, indeed, $A_{i}$ 's and $B_{i}$ 's may be 'far away' from $D$ in terms of edit distance (LEVENSHTEIN, 1966).

Thirdly, according to the previous description, the complexity of the translation method is basically quadratic in the size of the examples! Of course to reduce this complexity in our actual implementation, relevant pairs of sentences are selected on-the-fly according to some criterion. It suffices to say that in our current implementation, we do not inspect pairs $(A, B)$ where the length of $B$ is less than half that of $D$ or more than twice that of $D$ (and the same for $A$ relative to $B$ ). We shall not elaborate on this criterion as it is obvious that it is not optimal, neither theoretically, nor in terms of efficiency. It still remains to be determined what kind of criterion would be most efficient.

Fourthly, it follows from the algorithm above and the properties of proportional analogies that the method is non-deterministic. A plurality of translations may be obtained for one input sentence. This was made explicit in the algorithm above by the use of indices for $A$ 's, $B$ 's, $C$ 's and their counterparts in the target language, and also in the remarks in parentheses. Let us make it clear that the non-determinism of the method has four reasons.

1. many pairs $\left(A_{i}, B_{i}\right)$ can lead to a translation for $D$ (hence the use of $i$ to index these pairs);

2. analogical equations may have a plurality of solutions, so that any analogical equation $A_{i}: B_{i}:: x: D$ in the source language may yield several solutions (hence the introduction of index $j$ to denote such solutions: $\left.C_{i, j}\right)$;

3. each of $A_{i}, B_{i}$ and $C_{i, j}$ may have different translations, so that for any such source triple, there may be several corresponding analogical equations to solve in the target language (hence index $k)$;

4. again, as analogical equations may have a plurality of solutions, the analogical equations $\widehat{A}_{i}^{k}: \widehat{B}_{i}^{k}::{\widehat{C_{i, j}}}^{k}: y$ in the target language may yield different solutions (hence index $l$ for ${\widehat{D_{i, j}}}^{k, l}$ ).

Finally, it is necessary to mention that the same translation for the same input sentence may be output through different paths, i.e., 
different pairs $(A, B)$; different analogical equations $\quad A: B:: x: D$; different solutions to such analogical equations; different translations $\widehat{A}$ for $A, \widehat{B}$ for $B$, and $\widehat{C}$ for $C$; different analogical equations $\widehat{A}: \widehat{B}::$ $\widehat{C}: y$ and different solutions to such analogical equations. To sum it up, each different candidate translation $\widehat{D}$ output for $D$ may be assigned a number which is the number of times this particular $\widehat{D}$ was output. In Figures 5 and 6, where actual examples of translations are shown, these numbers are given at the left of each particular translation. It must be noted that these numbers are not small: in our experiments with a corpus of 160,000 aligned sentences, the same translation for the same input sentence may be output thousands of times. Currently, we poorly exploit these numbers, as we just consider that the most frequent translation should be the best one. This elementary criterion is used to select which translation candidate we use in evaluation with mWER, BLEU, NIST, etc.

\subsection{A Simple EXAMPle Without RECURSion}

To illustrate the method, suppose that we wanted to translate the following Japanese input sentence (gloss: strong coffee NOMINATIVEPARTICLE drink-VOLITIVE. Literally: I want to drink strong coffee.):

$$
D=\text { 濃いコーヒーが飲みたい。 }
$$

At some point in the exploration of all possible pairs of sentences from the bicorpus, we will find the following two Japanese sentences that litterally translate as Tea, please and Coffee, please but are actually translated as $\widehat{A}$ and $\widehat{B}$ in our database of examples:
(A) 紅茶をください。
$\leftrightarrow \quad(\widehat{A}) \begin{aligned} & \text { May I have some tea, } \\ & \text { please? }\end{aligned}$
(B) コーヒーをください。
$\leftrightarrow \quad(\widehat{B})$ May I have a cup of coffee?

Sentences $A$ and $B$ will allow us to form the following analogical equatior.

$(A)$ 紅茶をください。 $:(B) \begin{aligned} & \text { コーヒーを } \\ & \text { ください。 }:: ~ C ~\end{aligned}$

This equation yields $C=$ 濃い紅茶が飲みたい。 (lit.: I want to drink strong tea.). If this sentence already belongs to the bicorpus, i.e., if the following translation pair is found in the data 
$(C)$ 濃い紅茶が飲みたい。 $\quad \leftrightarrow \quad(\widehat{C}) \begin{aligned} & \text { I'd like some strong tea, } \\ & \text { please. }\end{aligned}$

the following analogical equation is formed with the corresponding English translations:

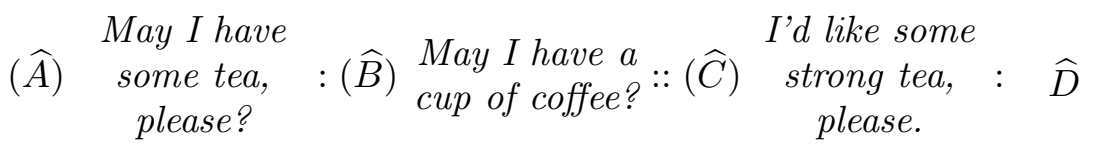

By construction, the solution: $\widehat{D}=I^{\prime} d$ like a cup of strong coffee. is a candidate translation of the input sentence: 濃いコーヒーが飲みたい。

The processing of the previous example can be viewed in the shape of a parallelopiped similar to the one of Figure 3. The left plane of this parallelopiped is the plane of the English analogy. The right plane is the Japanese one. The translation that was established is the one along the bottom front horizontal line.

$$
\begin{aligned}
& A=\begin{array}{r}
\text { May I have some } \\
\text { tea, pleäèe? }
\end{array} \\
& C=\begin{array}{c}
\text { I'd like some } \\
\text { strong tea a please. }
\end{array} \\
& B=\begin{array}{l}
\text { May I have a } \\
\text { cup of coffeè? }
\end{array} \\
& D=\begin{array}{c}
\text { I'd like a cup of } \\
\text { strong coffeee. }
\end{array}
\end{aligned}
$$

Figure 4. The parallelopiped for a translation from English into Japanese. $D$ is the input, $\widehat{D}$ is the output.

\subsection{ACtual EXAmples of translation}

As our motivation was to deal with translation divergences, Figure 5 illustrates the fact that some parts of speech in Japanese are rendered by different English parts of speech in actual translation results.

In the first example, a Japanese noun 料金 /ryōkin/ has been translated by verbs (cost, charge) in addition to being translated by nouns (price, fee) in different candidate translations. 
このツアーの料金はいくらですか。

/kono tuā no ryōkin wa ikura desu ka/

'this tour GEN fee/price (N) TOPIC how-much to-be INTERR'

271 How much does this tour cost?

160 How much do you charge for this tour?

141 What's the price of this tour?

94 What does this tour cost?

43 What's the price of the tour?

6 What is the price of the tour?

6 How much is the green fee?

\author{
胃が痛いんです。 \\ /i ga itai n desu/ \\ 'stomach NOM painful (A) INSIST to-be'
}

1744 I have a stomach ache.

552 My stomach hurts

124 I've got a stomach ache.

56 Do you have a stomach ache.

51 Do you have a stomach ache?

50 I have a stomach ache?

2 My stomach hurts me.

1 I have an abdominal pain in my stomach.

1 I have a pain in my stomach.

1 I have a soare throat.

Figure 5. Examples of translations. The numbers on the left are the frequencies with which each translation candidate has been output. Some parts of speech were changed during translation. In the first example, a Japanese noun $(\mathrm{N})$ appears to have been translated by verbs (cost, charge) in addition to being translated by nouns (price, fee) in other sentences. In the second example, a Japanese adjective (A) has been translated by a verb (hurts) or different nouns (ache, pain). Unacceptable translation candidates have been struck out. 
コーヒーのおかわりをいただけますか。

/kōhī no o-kawari wo itadakemasu ka/

'coffee GEN POLITE change/again ACC can-receive INTERR'

2318 I'd like another cup of coffee.

2296 May I have another cup of coffee?

1993 Another coffee, please.

1982 May I trouble you for another cup of coffee?

1982 Can I get some more coffee?

530 Another cup of coffee, please.

516 Another cup of coffee.

466 Can I have another cup of coffee?

337 May I get some more coffee?

205 May I trouble you for another cup of coffee, please?

小銭をまぜてください。

/kozeni wo mazete kudasai/

'coins/small-change ACC to-mix deign/if-you-please'

924 Can you include some small change?

922 Can you include some small change, please?

899 Would you include some small change?

896 Include some small change, please.

895 I'd like to have smaller bills mixed in.

895 Please change this into small money.

895 Will you include some small change?

885 Could you include some small change, please?

880 May I have some small change, too?

877 Please give me some small change as well.

Figure 6. Examples of translations. The numbers on the left are the frequencies with which each translation candidate has been output. 
In the second example, a Japanese adjective 痛い/itai/ has been rendered by a verb (hurts) or nouns (ache, pain). This example is the linguistic realisation of a statement about pain. In various languages, this kind of statement is realised in various ways, some of which can be schematised as follows (not an exhaustive list):

- $<$ body-part $><$ hurts $>[<$ somebody $>]$

- $[<$ somebody $>$-DAT $\mid<$ somebody $>$-GEN $]<$ body-part $><$ hurts $>$

- $[<$ there-is $>\mid<$ somebody $>$ has $]<$ pain $>$ in $<$ body-part $>$

The first form is used in the input sentence: 'stomach-NOM hurts' (where 'hurts' is expressed by an adjectival predicate). The second form is typical of Spanish: me duele la cabeza, la mano, ...., while the last one is usual to French: j'ai mal à la tête, à la main,... The translation example of Figure 5 shows that our system could synthesise some of these forms of expression for the sentence to be translated. Of course this was only possible because, the only knowledge of the system being the parallel corpus, realisations of such patterns were actually present in the corpus. What the example demonstrates is that the system could properly synthesise various forms of expression for the sentence to be translated because it could exploit actual realisations of such forms of expression and perform proper commutations. This demonstrates that actual unprocessed examples in conjunction with proportional analogy are at least as powerful as predefined patterns or templates with variable positions marked, like, say, I have $a<$ body part $>$ ache.

Moreover, such patterns are often too restricted for actual language usage, and they prevent the elastic use of idioms. Years ago, a former general in the French army wrote a book entitled J'ai mal à la France, something like: 'I have a France ache(?),' or 'France hurts me,' with the implied meaning that France was no external object for him, in contrast to a situation like: 'a needle hurts me.' A system where examples remain unprocessed has a greater chance of translating such an expression than a system where the previous pattern would be associated with a constraint restricting the choice to body parts.

\section{Theoretical foundations of the method}

This section gives some deeper insights into the theoretical aspects of the method proposed above. It should be mentioned that the previous method was in fact derived from the theoretical work that will now be described, not the contrary. 


\subsection{Proportional analogies between strings of CHARACTERS}

Our notion of analogies between sentences, or to be more precise between strings of characters, reaches back as far as Euclid and Aristotle: ' $A$ is to $B$ as $C$ is to $D$,' postulating the identity of types for $A, B$, $C$, and $D$. The notion was put forward in morphology by Apollonius Dyscolus and Varro in the Antiquity. In modern linguistics, Saussure (de SAussure, 1995, part. III, CHAP. IV) considers analogical equations as a typically synchronic operation by which, given two forms of a given word, and only one form of a second word, the fourth missing form is coined: 'honor is to honōrem as ōrātor is to ōrātōrem' (Latin: ōrātor (orator, speaker) and honor (honour) nominative singular, ōrātōrem and honōrem accusative singular):

ōrātōrem : ōrātor :: honōrem : $\quad \Rightarrow \quad x=$ honor

According to Saussure, this explains the fact that in the 2nd century $\mathrm{BC}$, the form honor actually competed with the etymologically correct form honos. While analogy has been largely mentioned and used in linguistics, only recently can we see applications of the notion in natural language processing to pronounciation, morphology or terminology: (Skousen, 1989), (Damper and Eastman, 1996), (Hathout, 2001), (Stroppa and Yvon, 2005), (Claveau and L'Homme, 2005), among other studies.

That analogy applies also to syntax, which is the foundation of our framework, has been advocated by Hermann Paul (PAUL, 1920, p. 110) and Bloomfield (Bloomfield, 1933, p. 275). More recently, Itkonen and Haukioja (ITKONEN and HAUkioJA, 1999) showed how to deliver grammatical sentences by application of proportional analogies to structural representations.

Algorithmic ways to solve proportional analogies between strings of characters have never been proposed, maybe because the operation seems so misleadingly 'intuitive.' An exception is Copycat (HoFSTADTER and the Fluid Analogies Research Group, 1994, p. 205-265), which adopts an artificial intelligence point of view, unfortunately of little use for linguistic applications that require very fast computation. To our knowledge, we were the first to give an efficient algorithm for the resolution of analogical equations in (LEPAGE, 1998) Our proposal is based on the following formalisation of proportional analogies (LEPAGE, 2003) in terms of edit distances, or equivalently, in terms of similarity (refer to (Stephen, 1994, Chap. 3) for these notions and see (Delhay and MiCLET, 2004) for an extension of this formalisation to alphabets equipped with an algebraic structure). We denote $\sigma(A, B, \ldots, N)$ as the 
length of the longest common subsequence in the strings $A, B, \ldots N$, i.e., their similarity. We also denote $|A|_{a}$ as the number of occurrences of character $a$ in string $A$ and $|A|$ as the length of $A$. The following formula consistently puts the unknown $D$ on the left of all equal signs, so as to better suit the resolution of analogical equations.

$$
A: B:: C: D \Rightarrow\left\{\begin{aligned}
\sigma(B, D) & =-|A|+|B|+\sigma(A, C) \\
\sigma(C, D) & =-|A|+|C|+\sigma(A, B) \\
\sigma(A, B, C, D) & =-|A|+\sigma(A, B)+\sigma(A, C) \\
|D|_{a} & =-|A|_{a}+|B|_{a}+|C|_{a}, \quad \forall a
\end{aligned}\right.
$$

As a remarkable property of proportional analogies, it is worth mentioning that the last relation, which expresses the fact that the number of occurrences of any character $a$ in $A$ and $D$ is equal to the number of its occurrences in $B$ and $C$, trivially implies that the lengths of the extremes ( $A$ and $D$ on one hand and $B$ and $C$ on the other hand) are equal:

$$
|A|+|D|=|B|+|C|
$$

For instance, applied to our example in the first sections:

$$
\begin{gathered}
\text { They swam } \begin{array}{c}
\text { It swam } \\
\text { in the sea. }
\end{array} \text { across the :: floated in : } \begin{array}{c}
\text { It floated } \\
\text { river. }
\end{array} \text { the sea. } \\
\text { river. }
\end{gathered}
$$

by counting characters we have (a space counts for one character):

$$
21+28=25+24
$$

The step-by-step mechanism we adopt during resolution is inspired by (ITKONEN and HAUKIOJA, 1999, p. 149), where they take sentence $A$ as the axis against which sentences $B$ and $C$ are compared, and by opposition to which output sentence $D$ is built.

Rather than explaining once again the algorithm given in (LEPAGE, 1998), we sketch its application in an actualised way on a particular analogical equation:

$$
\text { aslama : muslim :: arsala }: x \quad \Rightarrow \quad x=\text { mursil }
$$

In this Arabic example, arsala (he sent) and aslama (he converted [to Islam]) are 3rd person singular past verbs; mursil (a sender) and muslim (a convert, i.e., a muslim) are agent nouns.

For this sketch, we use words rather than sentences for reasons of space; the same algorithm applies to analogical equations between 
sentences considered as strings of characters; and it also applies to languages like Japanese, Chinese or Korean where a character is encoded by two bytes instead of just one byte for English.

We use (transliterated) Arabic words to show that solving analogical equations is not reduced to a trivial matter of exchanging prefixes or suffixes. In the morphology of Semitic languages, proportional analogies ought to capture parallel infixing (something that may help in the Arabic-English C-STAR track of IWSLT 2005, see Section 6.3). But more generally, parallel infixing is indispensable in our framework because proportional analogies between sentences involve parallel infixing in almost all of the cases.

As for the algorithm, pseudo-distance matrices between strings $A$ and $B$, and $A$ and $C$, are first computed. The pseudo-distance used here counts only insertions, and overlooks substitutions and deletions. A cell in a pseudo-distance matrix contains the value of the pseudodistance computed from the beginning of the strings up to the current positions. For instance, the cell with a value of 2 in boldface in the following example, gives the value of the pseudo-distance between the two prefixes of $C$ and $A$, ars and asla. To pass from ars to asla, it is necessary and sufficient to insert two symbols, $l$ and $a$, and to delete one: $r$. Deletions do not count, only insertions count, hence a value of 2. Based on the fast algorithm by (ALLISON and Dix, 1986) such a computation is performed in an efficient way. Also, a result by (UKKONEN, 1985) allows us to compute only minimal diagonal bands in those matrices.

The algorithm which computes the fourth term of the analogy follows all possible paths in parallel in the pseudo-distance matrices, in a way similar to that taken in (WAGNER and Fischer, 1974) for the output of edit distance traces. In the following example, a particular path is made explicit by circles, the indices of which indicate the move number. Paths start from the bottom of the matrices. Some constraints apply: circles with the same index must appear on the same line in both matrices; an arithmetic formula on the symbols in $A, B$ and $C$ must yield a result. Because of the constraints, paths may deviate from a trace in the sense of (WAGNER and Fischer, 1974). For instance, in the following example, move number 5 lands on 1 , not on the 0 to its right. 


$$
\begin{aligned}
& B=m \quad i \quad l \quad s \quad u \quad m \quad a \quad r \quad s \quad a \quad l \quad a=C \\
& \begin{array}{lllllllllllll}
1 & 1 & 1 & 1 & (1)_{6,7} & (1)_{8} & a & (1)_{6,7,8} & 0 & 0 & 0 & 0 & 0
\end{array} \\
& \begin{array}{lllllllllllll}
1 & 1 & 1 & \mathbb{1}_{5} & 2 & 2 & s & 1 & \mathbb{1}_{5} & 0 & 0 & 0 & 0
\end{array} \\
& \begin{array}{lllllllllllll}
1 & 1 & \text { (1) }_{4} & 2 & 3 & 3 & l & 2 & 2 & \text { (1) }_{4} & 1 & 0 & 0
\end{array} \\
& \begin{array}{lllllllllllll}
2 & (2) & 2 & 3 & 4 & 4 & a & 3 & 3 & \mathbf{2} & \text { (1) }_{3} & 1 & 0
\end{array} \\
& \begin{array}{lllllllllllll}
(2) & 3 & 3 & 4 & 4 & 4 & m & 4 & 4 & 3 & 2 & \text { (2) } 2 & 1
\end{array} \\
& \begin{array}{lllllllllllll}
(2) & 3 & 3 & 4 & 4 & 4 & a & 5 & 5 & 4 & 3 & 3 & \text { (2) } 1
\end{array} \\
& \text { A }
\end{aligned}
$$

The succession of moves is read in parallel in both matrices from the bottom to the top. Each move triggers the copies of characters into the solution $D$ (thus, in reverse order) according to 'rules' that tell which character to choose from which string $B$ or $C$ according to the different combinations of moves (diagonal, horizontal or vertical in each matrix). For instance, vertical moves forbid writing into $D$, two vertical moves are forbidden, etc. As a result, the solution $D=$ mursil is output as a possible solution for the analogical equation at hand.

\begin{tabular}{|c|c|c||c|c|}
\hline move $\#$ & $\operatorname{dir}_{A B}$ & $\operatorname{dir}_{A C}$ & copy onto $D$ & from string \\
\hline 1 & vertical & diagonal & $\epsilon$ & none \\
2 & diagonal & diagonal & $-m+m-l=l$ & $C$ \\
3 & diagonal & diagonal & $-a+i+a=i$ & $B$ \\
4 & diagonal & diagonal & $-l+l+s=s$ & $C$ \\
5 & diagonal & diagonal & $-s+s+r=r$ & $C$ \\
6 & horizontal & no move & $-a+u+a=u$ & $B$ \\
7 & no move & no move & $-a+m+a=m$ & $B$ \\
8 & & & & \\
\hline
\end{tabular}

In some cases, no path exists, which means that there is no solution to the analogical equation, and in general, several paths may exist so that there may be several solutions to an analogical equation. (Unfortunately, our formalisation of proportional analogies is yet to be completed, so that, with our implementation, we sometimes obtain more solutions than desired, but never less, at least as far as our experiments show on hundreds of linguistic examples in morphology and examples from formal languages.) 
Analogical equations are thus a ternary operation, i.e., a mapping $\alpha: \mathcal{L} \times \mathcal{L} \times \mathcal{L} \mapsto \wp(\mathcal{L})$ where $\wp(\mathcal{L})$ is the power set of $\mathcal{L}$ the set of strings considered. The set of the solutions of an analogical equation is:

$$
\alpha(A, B, C)=\{D \in \mathcal{L} \mid A: B:: C: D\}
$$

\subsection{LANGUAGES OF ANALOGICAL STRINGS}

Based on proportional analogies, we have shown (LEPAGE, 2001) how to define a family of formal languages, called languages of analogical strings. It is important to note that their construction does not make any use of non-terminals as is the case with simple contextual grammars (ILIE, 1998) (contextual grammars, not to be confused with contextsensitive grammars!). In fact, our proposal shares some aspects and concerns of contextual grammars.

Languages of analogical strings are built by transitive closure starting from a corpus of given sentences (strings of characters) $\Lambda_{0}$. We denote $\alpha(\Lambda, \Lambda, \Lambda)$ as the set of sentences produced by solving all possible analogical equations formed with three sentences in $\Lambda$.

$$
\alpha(\Lambda, \Lambda, \Lambda)=\left\{D \mid \exists(A, B, C) \in \Lambda^{3}, A: B:: C: D\right\}
$$

Then, the language $\mathcal{L}\left(\Lambda_{0}\right)$ of analogical strings built from a corpus $\Lambda_{0}$ is defined in the following way:

$$
\mathcal{L}\left(\Lambda_{0}\right)=\bigcup_{n=0}^{+\infty} \Lambda_{n} \text { where } \Lambda_{n+1}=\alpha\left(\Lambda_{n}, \Lambda_{n}, \Lambda_{n}\right)
$$

In fact, there is a chain of set inclusions $\Lambda_{n+1} \supset \Lambda_{n}$ because $\quad A: A::$ $A: x \quad \Rightarrow \quad x=A$.

As for the position of such languages in the Chomsky-Schützenberger hierarchy, it is easy to show that the classical regular language $\left\{a^{n} \mid n \geq 1\right\}$, the context-free language $\left\{a^{n} b^{n} \mid n \geq 1\right\}$, and the contextsensitive language $\left\{a^{n} b^{n} c^{n} \mid n \geq 1\right\}$ are all languages of analogical strings. Moreover, we have shown (LEPAGE, 2001) that the famous contextsensitive language $\left\{a^{n} b^{m} c^{n} d^{m} \mid m, n \geq 1\right\}$ used in (SHIEBER, 1985) to refute the context-freeness hypothesis of natural language, is a language of analogical strings. More importantly, every language of analogical strings meets the constant growth property, a property that intervenes partially in the definition of mild context-sensitivity, a notion introduced in (JosHI et al., 1991) to cope with the apparent power of human languages. 


\subsection{Homomorphisms Between languages of ANALOGical STRINGS}

The framework for translation by proportional analogies that we propose sees both the source and the target languages as languages of analogical strings that are defined from the set of sentences given in the parallel corpus. If we denote $\Lambda_{0}$ as the source language part of the parallel corpus and $\widehat{\Lambda_{0}}$ as its target language counterpart, we idealise the entirety of both source and target languages as being $\mathcal{L}\left(\Lambda_{0}\right)$ and $\mathcal{L}\left(\widehat{\Lambda_{0}}\right)$ according to the above notations.

Let us denote $\widehat{A}$ as the (set of) translations of a sentence $A$. The principle of translation is based on the following intuitive formula that is a transcription of the parallelopiped of Figure 4:

$$
A: B:: C: D \quad \Leftrightarrow \quad \widehat{A}: \widehat{B}:: \widehat{C}: \widehat{D} .
$$

Using the $\alpha$ operation that structures the source and target languages of analogical strings, an equivalent form of this formula is:

$$
\widehat{D}=\alpha(\widehat{A, B}, C)=\alpha(\widehat{A}, \widehat{B}, \widehat{C}) .
$$

This shows that this translation principle 'distributes' translation on the arguments of the structuring internal operation $\alpha$. Thus, it is a homomorphism between two languages of analogical strings that preserves the structuring operation, proportional analogy.

As we are concerned with translation divergences, and because divergences often imply different reorderings in different languages, let us add a word on this question and mention that the previous formalisation is indeed able to solve 'difficult' reordering problems. With its translation knowledge reduced to the two translation pairs: $a b c \leftrightarrow a b c$, $a b c a b c \leftrightarrow a a b b c c$, the system translates members of the regular language $\left\{(a b c)^{n} \mid n \in \mathbb{N}^{*}\right\}$ into the corresponding members of the context-sensitive language $\left\{a^{n} b^{n} c^{n} \mid n \in \mathbb{N}^{*}\right\}$, and reciprocally:

$$
(a b c)^{n} \leftrightarrow a^{n} b^{n} c^{n}
$$

by solving $2 \times(n-2)$ proportional analogies recursively.

\section{Features of the method}

\subsection{NO EXPLICIT TRANSFER}

To stress that the choice of a correct translation is really left to an implicit use of the structure of the target language, and does not imply 
any explicit transfer processing, let us consider the Spanish example of Section 2 again. The correspondences between the source and the target language in a proportional analogy will be entirely responsible not only for the selection of the correct lemmas wit their lexical POS, but also for the correct word order (see above for reordering).

The technique is also more general than the translation of the adnominal particle $N_{1}$ no $N_{2}$ from Japanese into English in (SumitA and IIDA, 1991) where the choice of the correct preposition (or word order) is left to the list of examples.

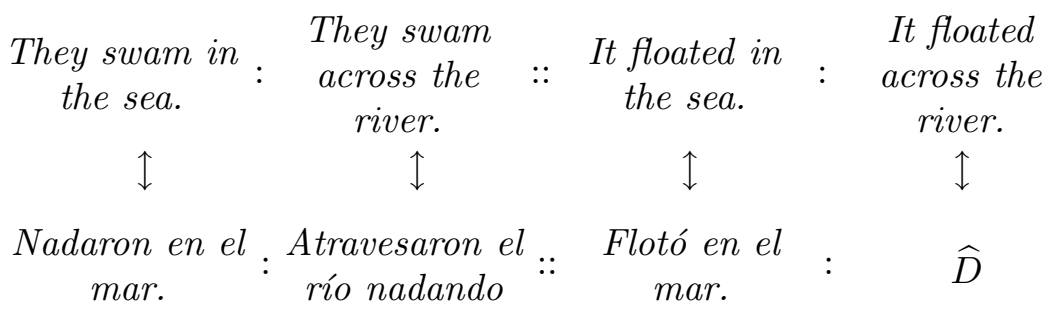

However, it should be stressed that in proportional analogies like the two above, nowhere is it said which word corresponds to which word, or which syntactic structure corresponds to which syntactic structure. To go back to the previous Spanish-English example on which we made explicit word-to-word correspondence, we stress again that in our method, the system does not see any sub-correspondence below that of the global correspondence between sentences. Hence, if we keep the same convention as before and put corresponding parts of the sentences into boxes, all that the system sees appears in fact as follows:

$$
\text { Atravesó el río flotando. } \leftrightarrow \text { It floated across the river. }
$$

i.e., the system sees only the entire correspondence between two sentences: a sentence in the source language corresponds to a sentence in the target language.

The sole action of proportional analogy with (necessarily) the character as the only unit of processing, is sufficient to produce the exact translation of It floated across the river, which is the correct Spanish sentence: $\widehat{D}=$ Atravesó el río flotando, provided that the three sentence pairs on the left are valid translation pairs.

\subsection{NO EXTRACTION OF SYMBOLIC KNOWLEDGE}

In a second generation MT system, one makes the knowledge relevant to such divergences explicit in the form of lexical and structural transfer rules. In the EBMT approach too, one makes this knowledge explicit 
by automatically acquiring templates that capture these divergences. In both cases, the knowledge about these divergences has to be made explicit. In our view, the choice of the correct expression ought to be left implicit as it pertains to the structure of the target language. Indeed, paradigmatic and syntagmatic commutations neutralise these divergences as they are the implicit constitutive material of proportional analogies.

Our system definitely positions itself in the EBMT stream, however it departs from it in one important aspect: it does not make any use of explicit symbolic knowledge such as templates with variables, and it does not produce any template either. Direct use of bicorpus data in their raw form is made, without any preprocessing.

The reason for doing so is that templates may well be insufficient in representing all of the implicit knowledge contained in examples. Indeed, variables in templates allow for paradigmatic variations at some predefined positions only. In (SATO, 1991), so as to acquire a grammar, sentences which differ by one word only are fed into a system. However, only regular languages can be learned by this technique.

For instance, extracting the template $X$ salts $Y$ from the example sentence the butcher salts the slice where $X$ may be replaced by the butcher, etc., and $Y$ by the slice, etc. (example taken from (CARL, 1998)), does not make the most of the potential of the example. Firstly, it prevents the butcher from being changed into a plural: the butchers. Moreover, it overlooks the fact that salts may also commute with its past and future forms, etc.: salted, will salt, etc., or with cuts, smokes, etc.; and so forth. To summarise, there is a risk of loss of information when replacing examples with templates.

The situation is in no way better with translation patterns. On the one hand, it is true that such translation patterns can be very efficiently indexed so that their retrieval is very fast. Super-functions introduced by (SASAYAMA et al., 2003) is such a means to extract and retrieve these kinds of translation associations using arrays. They make it explicit which variables in the source have to be replaced by which variables in the target. But it is well known that a single variable at one single position in a source template often needs to be linked to several positions distributed over a target template, and may even imply different levels of description (morphological, syntactical, etc.). For instance, negation is expressed at one single position in Japanese, whereas it may also imply a change in the form of the main verb in English: he eats $\rightarrow$ he does not eat.

Our view is that every position in a language datum is subject to paradigmatic variation. Putting it to the extreme, even phonetic variations have to be considered: wolf: wolves :: leaf: leaves. So that one 
definitely has to go below words. For this reason, our system processes strings of characters, not strings of words. The consequence is that a lot more exploitable information should be found in unprocessed examples than in templates. And it may well be the case that the templates necessary to encode the information contained in a set of examples are much larger in size than the actual size of these unprocessed examples themselves. Thus, extracting templates from examples may well entail a loss in generative power as well as in space. It must however be stressed that the generative power of the unprocessed examples does not actually reside in their bare listing but in their capacity to get involved in proportional analogies.

\subsection{No training, NO PREPROCESSING}

As a consequence of the above-mentioned features, there is no such thing as a training phase or a preprocessing phase in our system: the bicorpus is just loaded into memory at program start-up. No language model is computed; no alignment other than the one given by the bicorpus is extracted; no segmentation or tagging whatsoever is performed. Needless to say, the possibility of adding new information to the bicorpus is left open. For instance, adding dictionaries or paraphrases to the corpus is a possibility that may improve results but leaves the structure of the system absolutely unchanged (see Sections 6.4.2 and 6.4.3).

\section{Experiments, evaluation, comparison}

\subsection{Resources USED IN EVAluAtion}

To assess the performance of the proposed method, we used the CSTAR Basic Traveler's Expressions Corpus (http://www.c-star.org/). It is a multilingual resource of expressions from the travel and tourism domain that contains almost 160,000 aligned translations in Chinese, Korean, English and Japanese. In this resource, the sentences are quite short as the figures in the following table show. As the same sentence may appear several times with different translations, the number of different sentences in each language is indicated in the following table. 


\begin{tabular}{lrr}
\hline & $\begin{array}{c}\text { Number of } \\
\text { different sentences }\end{array}$ & \multicolumn{2}{c}{$\begin{array}{c}\text { Size in characters } \\
\text { avg. } \pm \text { std. dev. }\end{array}$} \\
\hline Chinese & 96,224 & $9.40 \pm 5.17$ \\
English & 97,395 & $35.17 \pm 18.83$ \\
Japanese & 103,051 & $16.22 \pm 7.84$ \\
Korean & 92,626 & $11.67 \pm 5.99$ \\
\hline
\end{tabular}

The method relies on the assumption that analogies of form are almost always analogies of meaning. Thus, prior to its application, we (LEPAGE, 2004) estimated the relative number of analogies of form which are not analogies of meaning in the resource used: less than $4 \%$ ( $\mathrm{p}$-value $=0.1 \%$ on a sample of 666 analogies). This proportion is too small to seriously endanger the quality of the results obtained during translation. An example of a false analogy, i.e., an analogy of form that is not valid is given below. It was extracted from the sampling set.

Could you tell Could you tell Where is the
me how to fill : me how to fill $\%$ conference : conference
this from. $\quad$ this form.
centre?

In the example above, the commutation between center and centre is just a matter of dialects of English, whereas the commutation between from and form is not acceptable, the first one being a spelling error.

\subsection{Comparison With Other Systems}

We assessed our system using the IWSLT 2004 tasks in both JapaneseEnglish and Chinese-English directions. As we used a bicorpus of 160,000 examples our results should be compared with those of the Unrestricted Data track reported in the proceedings of the evaluation workshop (AKIBA et al., 2004).

In this track, no restrictions were imposed on linguistic resources. As for tools, our system did not make any use of any NLP tool such as a tagger or the like to preprocess the data. In particular, we chose to place ourselves in the condition of standard natural Japanese and Chinese texts (in which no segmentation appears), so that we had to delete segmentation in the provided test sets! This clearly demonstrates that segmentation is not a necessity to perform a translation task from Japanese or Chinese. We consider that translation ought to be performed as much as possible on unmodified real texts without preprocessing as we want to evaluate machine translation systems, not preprocessing tools. As for data, no dictionary was used. The C-STAR 
Table I. Among the permitted resources, our system only used the C-STAR 160,000 aligned sentences. The IWSLT 2004 supplied corpus of 20,000 sentences is a subset of the C-STAR corpus, so that the other resources that our system used are just the remaining 140,000 sentences.

\begin{tabular}{lcc}
\hline \multicolumn{1}{c}{ Resources } & \multicolumn{2}{c}{ Data Track } \\
& Unrestricted & Our \\
& & configuration \\
\hline IWSLT 2004 corpus & yes & yes \\
\hline LDC resources & yes & no \\
\hline tagger & yes & no \\
\hline chunker & yes & no \\
\hline parser & yes & no \\
\hline external bilingual & yes & \\
dictionaries & & 140,000 additional \\
\hline other resources & yes & aligned sentences \\
\hline
\end{tabular}

corpus of around 160,000 aligned sentences described above was used for both language pairs. We refer to this as our 'training data', although there is absolutely no training phase within our framework. All these conditions are summarised in Table I.

In addition to the previous conditions, and in order to avoid the fact that some sentences in the test data may be included in the 'training data,' we assessed our system in two configurations: standard and open. The difference between the two is that, in the latter, any sentence from the test set was removed from the 'training data,' if found there.

Some examples of Japanese-English translations have already been given in Figures 5 and 6 . Let us recall that the numbers at the left of a translation candidate are the frequencies with which it has been output (see Section 3.1 and 3.2). As we assumed that the most frequent candidate should be the most reliable one, the evaluation was performed on the first candidates only.

Tables II and III summarize the evaluation results obtained with the objective criteria used in this evaluation campaign. The results for other systems were copied from (AKIBA et al., 2004, p. 11). The results 
Table II. Scores for the IWSLT 2004 Chinese-to-English Unrestricted Data track: no restriction on linguistic resources. The letters in indices at the left of the system names indicate their type: $s$ stands for statistical systems, $e$ for example-based systems, $r$ for rule-based systems, $h$ is for hybrid systems. Higher scores are better, except for mWER and PER, where lower scores indicate better results. In its open configuration our system tries to translate an input sentence again if it already belongs to the data, whereas in its standard configuration, it outputs the translation found in the data.

\begin{tabular}{lccccc}
\hline & mWER & mPER & BLEU & NIST & GTM \\
& & & & & \\
\hline${ }^{s}$ ISL-S & $\mathbf{0 . 3 7 9}$ & $\mathbf{0 . 3 1 9}$ & $\mathbf{0 . 5 2 4}$ & $\mathbf{9 . 5 6}$ & $\mathbf{0 . 7 4 8}$ \\
\hline${ }^{e}$ ours standard & 0.434 & 0.400 & 0.522 & 8.42 & 0.687 \\
\hline${ }^{e}$ ours open & 0.437 & 0.404 & 0.512 & 8.24 & 0.682 \\
\hline${ }^{s}$ IRST & 0.457 & 0.393 & 0.440 & 7.24 & 0.671 \\
\hline${ }^{s}$ IBM & 0.525 & 0.442 & 0.350 & 7.36 & 0.684 \\
\hline${ }^{h}$ ISL-E & 0.531 & 0.427 & 0.275 & 7.50 & 0.666 \\
\hline${ }^{s}$ ISI & 0.573 & 0.499 & 0.243 & 5.42 & 0.602 \\
\hline${ }^{h}$ NLPR & 0.578 & 0.531 & 0.311 & 5.92 & 0.563 \\
\hline${ }^{e}$ HIT & 0.594 & 0.487 & 0.243 & 6.13 & 0.611 \\
\hline${ }^{r}$ CLIPS & 0.658 & 0.542 & 0.162 & 6.00 & 0.584 \\
\hline${ }^{e}$ ICT & 0.846 & 0.765 & 0.079 & 3.64 & 0.386 \\
\hline
\end{tabular}

obtained for our system are very promising as our system achieves second place in Chinese-English, and third place in Japanese-English. A standout point is the achievement in BLEU: a close second for ChineseEnglish (0.522, first at 0.524), and the best one for Japanese-English (0.634). Unfortunately, we are not in a position to reproduce the subjective evaluation for the translation results output by our system. It must be stressed again that the above results were obtained without any training performed in advance on the data, and that no tuning whatsoever of the system towards the 'training data' was performed. 
Table III. Scores for the IWSLT 2004 Japanese-to-English Unrestricted Data track: no restriction on linguistic resources.

\begin{tabular}{lccccc}
\hline & mWER & mPER & BLEU & NIST & GTM \\
& & & & & \\
\hline \hline${ }^{h}$ ATR-H & $\mathbf{0 . 2 6 3}$ & $\mathbf{0 . 2 3 3}$ & 0.630 & 10.72 & 0.796 \\
\hline${ }^{s}$ RWTH & 0.305 & 0.249 & 0.619 & $\mathbf{1 1 . 2 5}$ & $\mathbf{0 . 8 2 4}$ \\
\hline${ }^{e}$ ours standard & 0.324 & 0.300 & $\mathbf{0 . 6 3 4}$ & 9.19 & 0.731 \\
\hline${ }^{e}$ ours open & 0.437 & 0.403 & 0.534 & 8.97 & 0.697 \\
\hline${ }^{e}$ UTokyo & 0.485 & 0.420 & 0.397 & 7.88 & 0.672 \\
\hline${ }^{r}$ CLIPS & 0.730 & 0.597 & 0.132 & 5.64 & 0.568 \\
\hline
\end{tabular}

\subsection{COMPARISON For Different language PAIRS}

Our system does not require any training phase so that data are merely loaded into memory before the system is made ready to translate. The IWSLT 2005 campaign offered a number of language pairs, with the possibility of using a multilingual corpus, where the amount and meaning of sentences are identical. We chose to participate in all C-STAR data tracks with exactly the same core engines in order to be able to compare the results obtained on different language pairs provided that the evaluation procedure was also the same. Our goal was to learn some lessons on the difficulty of translating some language pairs relatively to others with our proposed method. As only one configuration was allowed, we chose to use the open configuration of our system because it seemed the most honest attitude to inspect the potentialities of our method: whenever an input sentence was recognised as belonging to the training data, we excluded it from the database of translation pairs and tried to translate it anew. To do so seriously handicapped us, because such cases did actually occur. On 506 sentences to translate, 90 did in fact belong to the training set (and even to the supplied data of 20,000 sentences)! In an example-based system, by essence, such expressions should be translated by a mere memory access ${ }^{3}$.

Again as far as data are concerned, we intended to limit ourselves to the use of the core 160,000 C-STAR translation pairs. However, this was not possible for the Arabic-English track where only 20,000 translation 
Table IV. Scores for all IWSLT 2005 C-STAR tracks. Unless otherwise mentioned in Remarks, the system (open configuration) used the roughly 160,000 translation pairs of the C-STAR multilingual corpus in each language pair, and the evaluation was performed with 16 references.

\begin{tabular}{lcccccc}
\hline & mWER & mPER & BLEU & NIST & GTM & Remarks \\
\hline English-Chinese & 0.798 & 0.746 & 0.098 & 3.029 & 0.363 & 1 reference \\
\hline Arabic-English & 0.527 & 0.497 & 0.382 & 6.22 & 0.481 & 20,000 pairs \\
\hline Korean-English & 0.530 & 0.486 & 0.412 & 7.12 & 0.446 & \\
\hline Chinese-English & 0.454 & 0.418 & 0.477 & 7.85 & 0.553 & \\
\hline Japanese-English & 0.361 & 0.323 & 0.593 & 9.82 & 0.607 & \\
\hline
\end{tabular}

pairs were supplied. Consequently, a comparison of the Arabic-English results with other language pairs is not possible.

The results obtained are shown in Table IV. Again for all language pairs, no tool of any sort was used, which means that prior to translation, no segmentation or tagging whatsoever was performed. No dictionary was added to the corpus of example sentences. In fact, the results of our system should be considered as a sort of baseline for all these language pairs in the C-STAR tracks.

We have already said that because we used only 20,000 translation pairs in Arabic-English, we are not able to compare with other language pairs. We face another problem with the English-Chinese language pair: although the amount of data was 160,000 translation pairs as for other language pairs, evaluation was performed with only one reference whereas 16 references were used in all other pairs. It is well known that the number of references used enormously influences the scores in objective evaluation measures ${ }^{4}$. This prevents us from comparing the results.

To summarise, we are only able to conduct a comparison between the following language pairs: Korean-English, Chinese-English and Japanese-English. The scores obtained in these three language pairs may be compared because the amount of linguistic data used as examples does not change. Only the source language changes while the target language remains English in all cases with the very same examples. The results in all three main evaluation scores (mWER, BLEU and NIST) show that the performance of our system is lower for Korean- 
Table V. Number of analogies in the BTEC multilingual corpus.

\begin{tabular}{lrr}
\hline & $\begin{array}{c}\text { Number of } \\
\text { analogies }\end{array}$ & $\begin{array}{c}\text { Number of } \\
\text { sentences } \\
\text { involved }\end{array}$ \\
\hline English & $2,384,202$ & 53,250 \\
Japanese & $1,910,065$ & 53,572 \\
Chinese & $1,639,068$ & 49,675 \\
Korean & 266,504 & 25,088 \\
\hline
\end{tabular}

English whereas the best performance is achieved in Japanese-English, with Chinese-English being in the middle.

In both the IWSLT 2004 and IWSLT 2005 tasks, our system's scores are lower in the Chinese-English track than in the Japanese-English track, an observation which also holds true for the other competing systems. One could possibly infer that the Chinese data allow for fewer commutations than the Japanese data.

In the case of the Korean language, an issue is that of encoding. The hangul writing system uses one character to represent a syllable of the type CVC. Morphological commutations may take place whithin such a sequence. Relevant commutations should logically be sought at a scale lower than that of characters whereas we had our system working on the character level.

A more general interpretation of the results is that, in the view of our approach, the scores obtained by our system may well be interpreted as a measure of the 'systematicity' of the data contained in the linguistic resources used. In this view, our scores are consistent with the fact that the C-STAR BTEC is usually believed to be internally more homogeneous in Japanese than in Chinese, which is in turn usually believed to be more homogeneous than in Korean. This impression is confirmed by the statistics of Table $\mathrm{V}$, which gives the number of formal analogies present in each language part of the C-STAR BTEC. According to these statistics, Chinese exhibits fewer analogies than Japanese. In Korean, the number of sentences involved in at least one analogy is nearly half the number of sentences involved in other languages, which implies a much lower number of analogies in comparison with the other languages: roughly one eighth on average. There may be several reasons for this. Firstly, the Korean data may not be as homogenous and consistent as the other languages as they seem to have been produced by different people using quite different levels of 
Table VI. Scores for the Gold Standard, the baseline, and the system with various data. n.r. means not relevant.

\begin{tabular}{lcccccc}
\hline System: & $\begin{array}{c}\text { Number of } \\
\text { translation } \\
\text { pairs }\end{array}$ & mWER & mPER & BLEU & NIST & GTM \\
\hline Gold Standard & n.r. & 0.00 & 0.00 & 1.00 & 14.95 & 0.91 \\
\hline + Src + tgt paraph. & 438,817 & 0.46 & 0.42 & 0.50 & $\mathbf{8 . 9 8}$ & 0.67 \\
+ Tgt paraphrases & 318.668 & 0.47 & 0.43 & 0.49 & 8.91 & 0.67 \\
+ Src paraphrases & 369,822 & $\mathbf{0 . 3 8}$ & $\mathbf{0 . 3 5}$ & 0.53 & 8.53 & $\mathbf{0 . 6 8}$ \\
+ Dictionary & 206,382 & 0.39 & 0.36 & $\mathbf{0 . 5 4}$ & 8.54 & $\mathbf{0 . 6 8}$ \\
\hline Resource only & 158,409 & 0.39 & 0.36 & 0.53 & 8.53 & $\mathbf{0 . 6 8}$ \\
\hline 1/2 resource & 81,058 & 0.50 & 0.45 & 0.45 & 7.78 & 0.63 \\
1/4 resource & 40,580 & 0.53 & 0.49 & 0.42 & 7.18 & 0.60 \\
\hline Baseline: transl. memory 158,409 & 0.58 & 0.53 & 0.38 & 7.54 & 0.61 \\
\hline
\end{tabular}

language for similar situations. Secondly, as we said above, our method may miss commutations in Korean by relying on the character unit. Thirdly, and in accordance with the previous point, Korean is known to be much richer morphologically than Japanese or English (not to mention Chinese!) so that much more textual data should be logically needed to reflect the same amount of commutations in meaning.

\subsection{Choice And influence of the DAta}

In a third experiment, we evaluated the influence of adding or substracting data on the performance of our system. The test set used consists of 510 input sentences from the same domain as the bicorpus. Sixteen translation references in the target language were used for evaluation. As the data are all known to us in the experiment, we were able to determine a baseline and the upper bound for them.

The Gold Standard was determined in the following way. For each sentence of the test set, we evaluated the first reference translation as if it were given by an MT system. In this way, we obtained the 'best' values for each of the measures considered (see Table VI).

The baseline was determined by simulating a translation memory. For each sentence of the test set, we took the closest sentence in the corpus according to edit distance and output its translation, which 
we evaluated with each of the objective measures. This gives baseline scores for each of the measures considered.

Our system was then evaluated on its outputs for the sentences of the test set, with the sole resource of our 160,000 translation pairs (see Table VI, line: Resource only). Again, the evaluation was performed using the first candidates only, i.e., those with the highest output frequencies.

\subsubsection{Influence of the amount of examples}

In an EBMT system, one would trivially expect the amount and nature of examples to strongly influence translation quality. The figures in Table VI on the lines marked $1 / 2$ resource and $1 / 4$ resource confirm this fact. They were obtained by sampling the original resource. In this case, the more data, the better the results.

\subsubsection{Dictionaries as lists of particular examples}

Whole sentences contained in the resource (as opposed to isolated words or idioms) may not allow the translation of particular expressions if commutations cannot be found between them. This case is particularly plausible when translating sentences that contain multi-word expressions or numbers, for instance.

A possible remedy is to add dictionary entries to the original resource to be used as additional examples. As a matter of fact, in this system, there is no difference between a bicorpus or a dictionary as long as both are aligned strings of data, be they sentences or words. The following examples illustrate that the data format for a bicorpus or a dictionary does not differ in any way.

$\begin{array}{cccc}\text { フィルムを買いたいのですが。 } & \leftrightarrow & \text { I'd like a roll of film, please. } \\ \text { 三十六枚撮りを二本ください。 } & \leftrightarrow & \begin{array}{c}\text { Two rolls of thirty-six } \\ \text { exposure film, please. }\end{array} \\ \text { このカメラの電池がほしいのです。 } & \leftrightarrow & \text { I'd like a battery for this } \\ & & \text { camera, please. } \\ \text { フィルム } & \leftrightarrow & \text { film } \\ \text { 映画 } & \leftrightarrow & \text { film } \\ \text { 電池 } & \leftrightarrow & \text { battery } \\ \text { 砲台 } & \leftrightarrow & \text { battery }\end{array}$


The scores obtained by adding a dictionary to our resource are not different from those with the resource only, except for a slight improvement in BLEU.

6.4.3. Paraphrases generated from the resource as additional examples Previous research has shown that the introduction of paraphrases may improve the quality of machine translation output: paraphrases may be added in the source language (YАмамото, 2004) or in the target language (HABASH, 2002).

In order to increase the chances of a sentence entering into proportional analogies, we grouped sentences in the source language data by paraphrases. To do so, we grouped sentences that share at least one common translation because, in this case, they share the same meaning, (i.e., they are paraphrases). In our bicorpus, an average of 3.03 paraphrases per source sentence was obtained. However, the distribution is not uniform: 71,192 sentences (out of 103,274) don't get any new paraphrase, while 54 sentences get more than 100 paraphrases, with a maximum of 410 paraphrases for one sentence.

This new information allows the translation process to test a larger number of proportional analogies. When a pair of sentences $(A, B)$ is proposed for an input sentence $D$, not only will the equation $A: B:$ : $x: D$ be tried, but also all possible equations of the form $A^{\prime}: B^{\prime}::$ $x^{\prime}: D$, where $A^{\prime}$ and $B^{\prime}$ are paraphrases of $A$ and $B$.

The evaluation of translation quality when adding paraphrases in the source language are shown in Table VI on the line marked: + Src paraph. They show a slight improvement in word error rate.

The same thing can be done on the target language side with a similar effect of increasing the number of proportional analogies tried, this time in the target language. As for scores, they decrease in BLEU but show a real improvement in NIST.

The scores obtained when adding paraphrases in the source and in the target language are shown on the line marked: + Src + tgt paraph. They are not better than those with the resource only, except for NIST, as paraphrases are expected to have introduced lexical and syntactical variation in expressing identical meanings. An explanation for the loss in quality according to all other measures may be that the increase in computation may have overloaded the system (all experiments are done with the same time-out). 


\section{Discussion and future work}

\subsection{LEARNING AND LAZY PROCESSING}

In opposition to machine translation methods that "eagerly compile input samples and use only the compilations to make decisions" (AHA, 1998), our method "perform[s] less precompilation and use[s] the input samples to guide decision making". In this sense, the system presented here may be seen as a lazy learning system (AнA, 1997).

There is indeed an extra feature in our system: it learns as it keeps translating. As it appears from the description in Section 3.1, the system increases its knowledge by recursive calls because it adds new translation pairs to the bicorpus so that, in a normal setting, the history of translations influences the results of subsequent translations. However, in all experiments reported above we had to disable this feature so as to be placed in conditions comparable with, say, SMT systems. Of course, such a use denatures our system.

\subsection{Translation time}

It could have been feared that the complexity of the algorithm, which is basically quadratic in the amount of data, would have enormously impaired the method. However, using a simple heuristic (see Section 3.2, 'Thirdly...') to select only relevant pairs entering in analogical equations allowed us to keep translation times reasonable. Within a time-out of $1 \mathrm{CPU}$ second, the average translation time per sentence was 0.73 second on a $2.8 \mathrm{GHz}$ processor machine with $4 \mathrm{~Gb}$ memory.

\subsection{Proportion of Successful analogies}

As the fundamental operation in the system is analogy, we measured the proportion of analogical equations sucessfully solved over the total number of analogies formed in the source language. Between half a million and one million analogical equations $(687,641)$ are formed on average to translate one sentence from the test set. The proportion of analogical equations sucessfully solved is $28 \%$. In other words, in comparison with an ideal heuristic that would select only those pairs that lead to a solution, the current heuristic used to select sentence pairs from the corpus in order to form analogical equations is successful only a quarter of the time. Reaching $100 \%$ may be unattainable in practice but future work should include finding a heuristic that would increase this proportion so as to reduce the number of unnecessary trials. 


\subsection{RECURSION LEVEL NEEDED}

As was explained in Section 3.1, recursive applications are expected to be made in order to reach translations of a single input sentence. Over all input sentences of the test set, one recursive call is needed on average, and a maximum of two is necessary on some sentences. This shows that the sentences in the test set were in fact quite 'close' to the resource used: the number of recursive calls is a measure of how 'far' a sentence is from a corpus.

\subsection{Relevance / Suitability of the EXamples}

The translation of an input sentence depends crucially on the two following points. Firstly, whether the input sentence belongs to the domain (and the style) of the corpus of examples. Secondly, whether the corpus covers the linguistic phenomena present in the input sentence. A positive point of our system is that the absence of any training phase reduces the development cycle to the problem of choosing/coining suitable examples that cover a given domain and the linguistic phenomena of the language. To address these two issues, we see two possible directions of research.

Firstly, as was mentioned in Sections 6.4.3 and 6.4.2, we are studying various ways to add paraphrases or dictionaries and how to improve their efficiency in terms of lexical and syntactical variation, so as to further densify the bicorpus in terms of coverage.

Secondly, we are investigating the possibility of designing a core grammar by examples, i.e., a collection of examples that would cover the basic linguistic phenomena in a given language. In the same way as school grammars illustrate rules by examples, our methodology will be to choose a formal grammar known to have a large coverage, and to illustrate its rules with examples. Distributionalist grammars (HARRIS, 1982) seem to be better candidates for this purpose as they rely on the notion of the expansion and embedding of strings, a notion that is precisely captured by proportional analogy. In particular, string grammars (SAGER, 1981) or (SALKOFF, 1973) are well known for having a large coverage.

\section{Conclusion}

In this paper, we have shown that the use of a specific operation, namely proportional analogy, leads to reasonable results in machine translation without any preprocessing of the data whatsoever, an advantage over techniques requiring intensive preprocessing. In an experiment with 
a test set of 510 input sentences and an unprocessed corpus of almost 160, 000 aligned sentences in Japanese and English, we obtained BLEU, NIST and mWER scores of $0.53,8.53$ and 0.39 , respectively, well above a baseline simulating a translation memory. Slight improvements could be obtained by adding paraphrases.

The use of an operation that suits by essence the specific nature of linguistic data, i.e., their capacity for commutation on the paradigmatic and syntagmatic axes, allowed us to dispense with any preprocessing of the data whatsoever. In addition, this operation has the advantage of tackling the issue of divergences between languages in an elegant way: it neutralises them implicitly. As a consequence, the implemented system does not include any transfer component (either lexical or structural).

To summarise, we designed, implemented and assessed an EBMT system that, we think, can be dubbed the purest ever built as it strictly does not make any use of variables, templates or patterns, does not have any explicit transfer component, and does not require any training or preprocessing of the aligned examples, a knowledge that is, of course, indispensable. 


\section{Notes}

1 The confusion of analogy with mere similarity has its root in the scholastic elaboration of the notion. Seemingly, it originates in the writings of St Thomas of Aquinas and their interpretation by St Cajetan (who, nonetheless, duly acknowledged that the only rigorous acception of analogy is when one can say that $A$ is to $B$ as $C$ is to $D$ ). Boethius introduced a distinction between 'proportions' for ratios and 'proportionality' for an equality of ratios, i.e., an analogy. The recent work by Gentner and her colleagues (GENTNER, 1983) on what they call 'analogy' should rigorously be characterised as dealing with the fourth species of metaphors in Aristotle's definitions in the Poetics, i.e., those metaphors that are based on an analogy: 'an atom is like a solar system' because (and only because) 'an electron is to the nucleus as a planet is to the sun.'

2 One often generalises divergences across language families, by saying that motion verbs in Romance languages are usually translated into prepositions or verbal particles in Germanic and Slavic languages. Hence, one would oppose the series:

- Fr. il traversa la rivière à la nage

- It. ha attraversato nuotando il fiume

- Sp. atravesó el río nadando

to their Germanic or Slavic counterparts:

- En. he swam across the river

- Ger. er durch schwamm den Fluss

- Pol. przeptywat przez rzeke

A remark on this last example. Although attested on the Web (argumentum ad Gogulum!), the verb przeptynać / przeptywać does not appear in the Stownik syntaktycznogeneratywny czasowników polskich (Syntax generative dictionary of Polish verbs), K. Polański, ed., Wrocław 1984. In our opinion, this 'latency' is a testimony of the productivity of such morphological constructs.

3 In participating in IWSLT 2005, we wanted to demonstrate the potentialities of our approach, rather that obtaining the best possible numerical results. Our real goal was to compare results obtained in different language pairs. It would have been trivially possible for us to get excellent results with our system using a standard configuration and suitable data. Indeed the ultimate essence of an example-based system is to comprise a translation memory and this is the case with our system. The track we participated in was the so-called C-STAR data track for which it was formally specified that: '[t]here are no limitations on the linguistic resources used to train the MT systems. Full BTEC corpus and proprietary data can be used.' Almost all the test sentences could be found in our proprietary data, so that, with the standard configuration of our system, such data, and a minimum of computation, we actually got the following scores in a 'false' run: $\mathrm{mWER}=0.07, \mathrm{BLEU}=0.93$ and NIST $=14.13$.

4 An epistemological remark: mWER, BLEU, NIST and the like are often said to be measures for translation. Strictly speaking, this is not true. They are just families of measures. Only the given of the BLEU (NIST, ...) formulae plus references constitute a measure, not the BLEU (NIST, ...) formulae alone. 


\section{Authors' Vitae}

\section{Y. Lepage}

This author holds a Ph.D. in computer science (specialisation: computational linguistics) received from Grenoble University, GETA, in 1989. He defended a habilitation thesis in 2003 at the same university on the subject: 'Of that kind of analogies capturing linguistic commutations' (De l'analogie rendant compte de la commutation en linguistique). Until April 2006, he was a senior researcher at ATR Spoken Language Communications Laboratories, Keihanna, Japan. From this date on, he is with the Japanese National Institute of Information and Communications Technology (NiCT). He is a member of the French and the Japanese Associations for Natural Language Processing, ATALA and Gengo syori gakkai.

\section{E. Denoual}

Until April 2006, this author was a researcher at ATR Spoken Language Communications Laboratories, Keihanna, Japan. From this date on, he is with the Japanese National Institute of Information and Communications Technology $(\mathrm{NiCT})$. He is a Ph.D. candidate at Joseph Fourier University, and a member of GETA-CLIPS-IMAG, Grenoble, France.

\section{References}

Aна, D. W.: 1997, 'Editorial'. Artificial Intelligence Review, Special Issue on Lazy Learning. 11(1-5), 7-10.

AнA, D. W.: 1998, Feature Weighting for Lazy Learning Algorithms. In: (LiU and MоTOD, 1998).

Akiba, Y., M. Federico, N. Kando, H. Nakaiwa, M. Paul, and J. Tsujis: 2004, 'Overview of the IWSLT04 Evaluation Campaign'. In: Proc. of the International Workshop on Spoken Language Translation. Kyoto, Japan, pp. 1-12.

Allison, L. and T. I. Dix: 1986, 'A bit string longest common subsequence algorithm'. Information Processing Letter 23, 305-310.

Amores, J. G. and J. P. Mora: 1998, Machine Translation of Motion Verbs from English to Spanish. In: (MARTín-VIDE, 1998).

Bloomfield, L.: 1933, Language. New York: Holt.

Brown, P. E., V. J. Della Pietra, S. A. Della Pietra, and R. L. Mercer: 1993, 'The Mathematics of Statistical Machine Translation: Parameter Estimation'. Computational Linguistics, Special Issue on Using Large Corpora: II 19(2), 263311.

CARL, M.: 1998, 'A constructivist approach to machine translation'. In: D. PowERS (ed.): Proceedings of NeMLaP'98 / CoNLL98: New Methods in Language Processing and Computational Natural Langugae Learning. Sydney, pp. 247-256.

CARL, M. and A. WAY: 2003, Recent advances in Example-based machine translation, Text, Speech and Technology. Dordrecht: Kluwer Academic Publishers. 
Claveau, V. and M.-C. L'Homme: 2005, 'Apprentissage par analogie pour la structuration de terminologie - Utilisation comparée de ressources endogènes et exogènes'. In: Actes de Terminologie et intelligence artificielle. Université de Rouen.

Damper, R. I. and J. E. Eastman: 1996, 'Pronouncing Text by Analogy'. In: Proceedings of COLING-96. København, pp. 268-269.

de Saussure, F.: 1995, Cours de linguistique générale. Lausanne et Paris: Payot.

Delhay, A. and L. Miclet: 2004, 'Analogical Equations in Sequences: Definition and Resolution'. Lecture Notes in Computer Science 3264, 127-138.

Dorr, B. J., L. Pearl, R. Hwa, and N. Habash: 2002, 'DUSTer: A Method for Unraveling Cross-Language Divergences for Statistical Word-Level Alignment'. In: Proceedings of the Fifth Conference of the Association for Machine Translation in the Americas (AMTA-2002).

Gentner, D.: 1983, 'Structure Mapping: A Theoretical Model for Analogy'. Cognitive Science 7(2), 155-170.

Habash, N.: 2002, 'Generation-Heavy Hybrid Machine Translation'. In: Proceedings of the International Natural Language Generation Conference (INLG'02). New York, pp. 185-191.

HARRIS, Z.: 1954, 'Distributional structure'. Word 10, 146-162.

HARRIS, Z.: 1982, A grammar of English on mathematical principles. New York: John Wiley \& Sons.

Hathout, N.: 2001, 'Analogies morpho-synonymiques. Une méthode d'acquisition automatique de liens morphologiques à partir d'un dictionnaire de synonymes'. In: Actes de TALN-2001. Tours, pp. 223-232.

Hofstadter, D. and the Fluid Analogies Research Group: 1994, Fluid Concepts and Creative Analogies. New York: Basic Books.

Ilie, L.: 1998, On Ambiguity in Internal Contextual Languages, pp. 29-45. in (MARTÍN-VIDE, 1998)

ItKonen, E. and J. HAUKIOJA: 1999, Grammaticalization: Abduction, Analogy, and Rational Explanation, pp. 159-175.

Joshi, A., K. ViJay-Shanker, and D. Weir: 1991, The Convergence of Mildly Context-Sensitive Grammar Formalisms, pp. 31-81. In: (SELls et al., 1991).

Lepage, Y.: 1998, 'Solving Analogies on Words: an Algorithm'. In: Proceedings of COLING-ACL'98, Vol. I. Montreal, pp. 728-735.

Lepage, Y.: 2001, 'Analogy and formal languages'. In: Proceedings of FG/MOL 2001. Helsinki, pp. 1-12.

LEPAGE, Y.: 2003, 'De l'analogie rendant compte de la commutation en linguistique'. Mémoire d'habilitation à diriger les recherches, Université de Grenoble.

LePAGE, Y.: 2004, "Lower and higher estimates of the number of "true analogies" between sentences contained in a large multilingual corpus'. In: Proceedings of COLING-2004, Vol. 1. Genève, pp. 736-742.

Lepage, Y. and G. Peralta: 2004, 'Using paradigm tables to generate new utterances similar to those existing in linguistic resources'. In: Proceedings of LREC-2004, Vol. 1. Lisbonne, pp. 243-246.

Levenshtein, V.: 1966, 'Binary codes capable of correcting deletions, insertions and reversals'. Soviet Physics-doklady 10(8), 707-710.

Liu, H. and H. MотоD: 1998, Feature Extraction, Construction and Selection: A Data Mining Perspective. Norwell MA: Kluwer.

MARTín-VIDE, C.: 1998, Mathematical and computational analysis of natural language. Amsterdam / Philadelphia: John Benjamins Publishing Co.

Paul, H.: 1920, Prinzipien der Sprachgeschichte. Tübingen: Niemayer. 
SAGER, N.: 1981, Natural language information processing: a computer grammar of English and its applications. Massachusetts: Adelson-Wesley, Reading.

SAlKoff, M.: 1973, Une grammaire en chaîne du français. Paris: Dunod.

Sasayama, M., F. Ren, and S. Kuroiwa: 2003, 'Super-function based JapaneseEnglish machine translation system'. In: Proceedings of Natural Language Processing and Knowledge Engineering, Vol. 1. Beijing, pp. 555-560.

SAto, S.: 1991, 'Example-based Machine Translation'. Ph.d. thesis, Kyoto University.

Sells, P., S. Shieber, and T. Wasow (eds.): 1991, Foundational issues in natural language processing. Cambridge: MIT Press.

Shieber, S. M.: 1985, 'Evidence against the Context-Freeness of Natural Language'. Linguistics and Philosophy 8, 333-343.

Skousen, R.: 1989, Analogical modeling of language. Dordrecht: Kluwer.

Stephen, G. A.: 1994, String searching algorithms. Singapore New Jersey London Hong Kong: World scientific.

Stroppa, N. and F. Yvon: 2005, 'An analogical learner for morphological analysis'. In: Proceedings of the 9th Conference on Computational Natural Language Learning (CoNLL 2005). Ann Arbor, MI, pp. 120-127.

Sumita, E.: 2003, EBMT using DP-matching between word sequences, pp. 189-209. In: (CARL and WAY, 2003).

Sumita, E. and H. IIDA: 1991, 'Experiments and Prospects of Example-Based Machine Translation'. In: Proceedings of the 29th Conference on Association for Computational Linguistics. Morristown, NJ, USA, pp. 185-192.

UkKonen, E.: 1985, 'Algorithms for Approximate String Matching'. Information and Control 64, 100-118.

Wagner, R. A. and M. J. Fischer: 1974, 'The String-to-String Correction Problem'. Journal for the Association of Computing Machinery 21(1), 168-173.

Wu, D.: 1997, 'Stochastic Inversion Transduction Grammars and Bilingual Parsing of Parallel Corpora'. Computational linguistics 23(3), 377-403.

Yамамото, K.: 2004, 'Interaction between paraphraser and transfer for spoken language translation'. Journal of Natural Language Processing 11(5), 63-86.

Address for Offprints: ATR Spoken Language Communication Research Labs, Keihanna, Hikari-dai 2-2-2, 619-0288 Kyoto, Japan 